\title{
On the feasibility of the detection of differential rotation in stellar absorption profiles
}

\author{
A. Reiners ${ }^{1}$ and J. H. M. M. Schmitt ${ }^{1}$ \\ Hamburger Sternwarte, Universität Hamburg, Gojenbergsweg 112, 21029 Hamburg, Germany
}

Received 24 September 2001 / Accepted 12 December 2001

\begin{abstract}
Stellar differential rotation invokes subtle effects on line absorption profiles which can be best studied in the Fourier domain. Detailed calculations of the behavior of Fourier transformed profiles with respect to varying differential rotation, limb darkening and inclination angles are presented. The zero positions of the Fourier transform are found to be very good tracers of differential rotation. The ratio of the first two zero positions $\sigma_{2} / \sigma_{1}$ can be easily measured and is a reliable parameter to deduce the amount of differential rotation. It is shown that solar-like differential rotation (equatorial regions have larger angular velocity then polar regions) has an unambigious signature in the Fourier domain and that in certain cases it can easily be distinguished from limb darkening effects. A simple procedure is given allowing the determination of the amount of differential rotation by the knowledge of the first two zero positions of a line profile's Fourier transform alone (i.e., without the need for thorough atmospheric modelling), under the assumption of a linear limb darkening law with a limb darkening coefficient of $\epsilon=0.6$.
\end{abstract}

Key words. stars: rotation - line: profiles

\section{Introduction}

Differential rotation is a central ingredient of the general accepted stellar activity paradigm, according to which a magnetic dynamo is ultimately responsible for the plethora of observed activity phenomena. Model calculations of stellar dynamos including differential rotation have been carried out (e.g. Kitchatinov \& Rüdiger 1999) but only a few measurements of stellar differential rotation exist.

Three approaches to determine differential rotation exist: (a) By identifying individual features on Doppler maps and following their migration with time; (b) by studying the rotation law with time; and (c) by studying line profiles. Method (a) has been used for example for AB Dor (Donati \& Collier Cameron 1997), PZ Tel (Barnes et al. 2000) and the rapidly rotating giant KU Pegasi (Weber \& Strassmeier 2001). At least two different images of the surface of the star are necessary to draw conclusions about differential rotation by this method. The construction of two (or more) Doppler images requires good phase coverage with high signal to noise; consequently large amounts of observing time are needed. For method (b) it is assumed

Send offprint requests to: A. Reiners, e-mail: areiners@hs.uni-hamburg.de that activity regions dominating the rotational period migrate in latitude over the stellar surface during a magnetic cycle and thus lead to an apparent change in rotation rate. The observations must cover at least a complete magnetic cycle, which makes these projects difficult and time consuming, too. For method (c), which we want to revisit in this paper, only one single exposure with large spectral resolution and high signal to noise is needed. However, to our knowledge only one successful measurement (Reiners et al. 2001) of non-rigid rotation through line profile analysis exists.

The possibility of detecting differential rotation through line profile analysis is discussed in a serie of publications (Huang 1961; Gray 1977; Bruning 1981; GarcíaAlegre et al. 1982), but the extent of these studies is limited to only a few cases, which do not provide a consistent overall picture. Furthermore, differences between the calculations are mentioned which can only partly be explained by the different underlying assumptions (see also Bruning 1982). In principle, a search for differential rotation effects can be carried out on every line profile measured with high signal to noise, however, in order to decide whether rigid rotation is consistent with the data or not a complete atmospheric model including all atomic data, turbulence and geometric effects must be carried out. 
This is rather cumbersome and no convenient observable is tabulated for a quick check on whether a star is differentially rotating or not.

The purpose of this paper is to revisit the effects of differential rotation on absorption line profiles. Detailed calculations are presented which in particular do allow a clear separation of the included model parameters. In particular we present tabulated observables for a quick and easy check on whether a line profile is consistent with rigid rotation or not. Thus large samples of stars can be analysed for differential rotation effects without the need to carry out a time-consuming line profile modelling for every object.

\section{The Fourier-transformed profile}

The basic assumption of our approach is to interpret a given absorption line profile $D(\lambda)$ as a convolution (denoted by $*$ ) between an "intrinsic" line profile $F(\lambda)$ which here is the line profile including atomic data (e.g. damping coefficients), temperature and element abundance effects, turbulent velocity fields and instrumental effects - and a rotational broadening function $G(\lambda)$ including limb darkening. With this assumption $D(\lambda)$ can be written as

$$
D(\lambda)=F(\lambda) * G(\lambda) .
$$

It is further assumed that the line profiles are identical over the stellar surface including the deeper layers. The validity of this assumption is not obvious and one has to check individual cases, but particularly for fast rotators rotation dominates the line profiles and our assumption becomes more and more reliable. Gray (1976) also developed broadening functions inferred from turbulent velocity fields. In this paper we show that, except for very slow rotators, the complete modeling of $D(\lambda)$ is not necessary for a determination of differential rotation.

For the analysis of absorption lines, Fourier transform of the profiles is convenient because Eq. (1) simplifies in Fourier domain to

$d(\sigma)=f(\sigma) \cdot g(\sigma)$,

where we use the Fourier frequency $\sigma$ expressed in cycles $/\left(\mathrm{km} \mathrm{s}^{-1}\right)=\mathrm{s} \mathrm{km}^{-1}$ and $d(\sigma), f(\sigma)$ and $g(\sigma)$ are the Fourier transforms of $D(\lambda), F(\lambda)$ and $G(\lambda)$, respectively. In the Fourier domain convolutions become multiplications. Therefore any zero in the Fourier transformed rotational broadening function $g(\sigma)$, which contains the information on differential rotation, must also appear in the observed Fourier transform $d(\sigma)$. The latter can be observed in the transformed data profiles without any data manipulation and in particular without any deconvolution of $F(\lambda)$.

For rigid rotation the zeros in $g(\sigma)$ can be analytically calculated. Using the limb darkening law

$$
I_{\lambda}(\theta) / I_{\lambda}(0)=1-\epsilon+\epsilon \cos \theta
$$

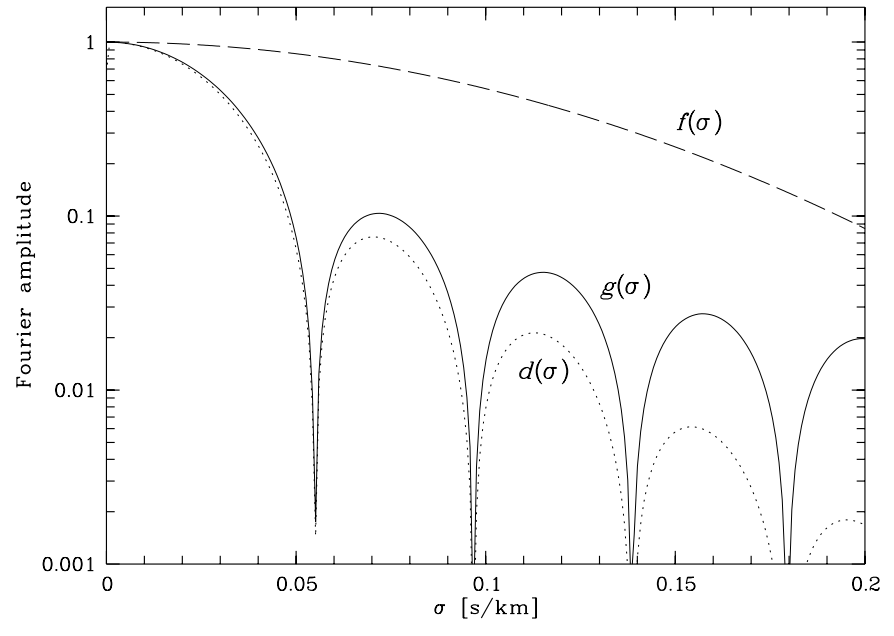

Fig. 1. A typical normalized Fourier transformed rotational broadening function $g_{\epsilon}(\sigma)\left(v \sin i=12 \mathrm{~km} \mathrm{~s}^{-1}\right.$; solid line) and the Fourier transform of a Gaussian broadening function $f(\lambda)$, (e.g. isotropic turbulence) with $v_{\text {iso }}=5 \mathrm{~km} \mathrm{~s}^{-1}$ (dashed line). The multiplication $d(\sigma)=f(\sigma) \cdot g(\sigma)$ is shown with a dotted line.

where $I_{\lambda}(\theta)$ denotes the intensity from a surface element, whose angle between its normal and the observer's line of sight is given by $\theta$. The limb darkening dependent rotationally broadening profile centered at some wavelength $\lambda_{0}$ can be expressed as

$$
\begin{aligned}
& G_{\epsilon}\left(\lambda-\lambda_{0}\right)= \\
& \quad \frac{2(1-\epsilon)\left(1-\left(\frac{\lambda-\lambda_{0}}{\delta}\right)^{2}\right)^{\frac{1}{2}}+\frac{\pi \epsilon}{2}\left(1-\left(\frac{\lambda-\lambda_{0}}{\delta}\right)^{2}\right)}{\pi \delta\left(1-\frac{\epsilon}{3}\right)}
\end{aligned}
$$

where $\left|\lambda-\lambda_{0}\right|<\delta$ and $\delta=\left(\lambda_{0} v \sin i\right) / c$ (cf. Unsöld 1968; Gray 1976). In terms of the variable $x=2 \pi \delta \sigma$ the Fourier transform of Eq. (4) is given by Böhm (1952) as:

$g_{\epsilon}(\sigma)=\frac{2}{x\left(1-\frac{\epsilon}{3}\right)}\left[(1-\epsilon) J_{1} x+\epsilon\left(\frac{\sin x}{x^{2}}-\frac{\cos x}{x}\right)\right]$,

with $J_{1}(x)$ denoting the first order Bessel-function. The $\epsilon$ dependent zeros of $g_{\epsilon}$ are therefore determined from the equation

$(1-\epsilon) J_{1} x+\epsilon\left(\frac{\sin x}{x^{2}}-\frac{\cos x}{x}\right)=0$.

An example of a typical normalized Fourier transformed rotational broadening function $g_{\epsilon}(\sigma)$ is shown in Fig. 1. The "main lobe" centered on $\sigma=0$ is followed by a series of "sidelobes". Important attributes are the zero positions $\sigma_{1}, \sigma_{2}$ etc. and the sidelobe amplitudes $I_{1}, I_{2}$ etc. The dashed line shows the Fourier transform of an assumed intrinsic Gaussian line profile with a dispersion of $v_{\text {disp }}=5 \mathrm{~km} \mathrm{~s}^{-1}$. The Fourier transformed data profile $d(\sigma)=g(\sigma) \cdot d(\sigma)$ is shown with a dotted line. Since in our specific case the Fourier transformed broadening function $f(\sigma)$ decreases monotonically, the amplitudes of the 
sidelobes especially at higher frequencies $\left(I_{2}, I_{3} \ldots\right)$ are affected by the multiplication with $f(\sigma)$ while the zero positions of $g(\sigma)$ remain unchanged and their determination require no knowledge of $f(\sigma)$.

$g_{\epsilon}(\sigma)$ and its zero positions $\sigma_{n}$ scale with $v \sin i$ (Carroll 1933a; Carroll 1933b); $\sigma_{n}$ can be expressed as

$\sigma_{n}=q_{n}(\epsilon) / v \sin i$.

Thus, given $\epsilon, v \sin i$ can directly be determined from the zero positions of $d(\sigma)$. Dravins et al. (1990) found an approximation for $q_{1}(\epsilon)$ and $q_{2}(\epsilon)$ :

$$
\begin{aligned}
q_{1} & =0.610+0.062 \epsilon+0.027 \epsilon^{2}+0.012 \epsilon^{3}+0.004 \epsilon^{4} \\
q_{2} & =1.117+0.048 \epsilon+0.029 \epsilon^{2}+0.024 \epsilon^{3}+0.012 \epsilon^{4} \\
\frac{q_{2}}{q_{1}} & =1.831-0.108 \epsilon-0.022 \epsilon^{2}+0.009 \epsilon^{3}+0.009 \epsilon^{4}
\end{aligned}
$$

and thus

$$
1.72<\frac{q_{2}}{q_{1}}<1.83 \quad(0.0<\epsilon<1.0) .
$$

For a parameterization of differential rotation we use a rotation law analogous to the solar case:

$\omega(l)=\omega_{0}-\omega_{1} \sin ^{2} l$,

with $l$ denoting latitude. Specifically, differential rotation is expressed in terms of $\alpha$,

$\alpha=\frac{\omega_{1}}{\omega_{0}} \quad\left(\alpha_{\odot}=0.20\right)$

$\alpha=0$ means solid body rotation, $\alpha>0$ equatorial acceleration (solar-like differential rotation) and $\alpha<0$ polar acceleration.

Many efforts have been undertaken to find a parameterization of $g_{\epsilon, \alpha}(\sigma)$ for the case of a differentially rotating star. Huang (1961) found solutions for special cases $\left(i=90^{\circ}, \epsilon=0.0\right)$ but no analytical forms for the general case are known. Thus modeling of differential rotation has to be performed by numerical integration over the stellar surface. Gray (1977) examined the equator-on case $\left(i=90^{\circ}\right)$ and found that the ratio of first to second sidelobe amplitudes is smaller in case of solar-like differential rotation $(\alpha>0)$. Bruning (1981) and García-Alegre et al. (1982) calculated profiles for differentially rotating stars; Bruning's calculations assume $\epsilon=0.6$ while GarcíaAlegre et al. neglected limb-darkening $(\epsilon=0.0)$. Both authors also investigated a few cases with $i<90^{\circ}$ and found differences to the equator-on case. Substantial differences exist between the mentioned calculations, which Bruning (1982) attributed to the different values of the limb darkening parameter $\epsilon$ used. Furthermore, although Bruning (1981) did not directly mention the amplitude of the second sidelobe, his Fig. 4 is inconsistent with the calculations of García-Alegre et al. (1982). Bruning's Table 1 has been the reference for analyses e.g. by Gray (1982).

We thus conclude that previous calculations do not show a clear picture of the important parameter dependences of differential rotation. No approximations for
Eqs. (8)-(10) with $\alpha$ included are known. We therefore carried out detailed calculations of the changes of Fourier transformed line profiles with differential rotation, and especially focused on the inclination dependence and the possibility of distinguishing limb darkening effects from differential rotation effects.

\section{Model}

Let us first consider the rotational broadening function $G(\lambda)$ for a differentially rotating star. For the case of rigid rotation $G(\lambda)$ can be expressed analytically as in Eq. (4), to numerically calculate $G(\lambda)$ for the case of differential rotation we use a modified version of the package developed and described by Townsend (1997). The rotation law (12) and limb darkening law (3) was applied. The integration is carried out over 25500 visible surface elements. To reduce numerical noise we used a Gaussian profile as input function instead of a $\delta$-function. This is equivalent to the convolution of a Gaussian profile $F(\lambda)$ with the desired rotational broadening profile $G(\lambda)$. We chose an equivalent width of $1 \AA$ for the Gaussian input function, which implies that the Fourier transformed profile is normalized to amplitude 1 at the abscissa. Similar to the convolution of the rotational broadening profile $g(\sigma)$ with the intrinsic line broadening profile $f(\sigma)$ discussed in Sect. 2, our specifically chosen input function affects the amplitude of $g(\sigma)$ and has to be taken into account when amplitudes of $g(\sigma)$ are considered. However, in our case the Fourier transformed Gaussian input profile has an amplitude of $99.93 \%$ at $0.2 \mathrm{~s} \mathrm{~km}^{-1}$; since our study focuses on the region $\sigma<0.15 \mathrm{~s} \mathrm{~km}^{-1}$, we applied no correction to $g(\sigma)$.

We arbitrarily centered the input function at $\lambda_{0}=$ $6251.826 \AA$. We chose a spectral resolution of $0.003 \AA$ $\left(0.14 \mathrm{~km} \mathrm{~s}^{-1}\right)$ and used a grid of 8192 points on which Fourier components were computed. The calculated profiles depend on four parameters, the differential rotation $\alpha$, the limb darkening coefficient $\epsilon$, equatorial rotational velocity $v_{\text {eq }}$ and the inclination $i$ of the rotation axis.

\section{Results}

In Figs. 2-4 we show the dependence of the absorption line profiles (left panel) and the corresponding Fourier transforms (right panel) for a projected rotational velocity of $v \sin i=12 \mathrm{~km} \mathrm{~s}^{-1}$ on $\alpha, \epsilon$ and $i$. Figure 2 shows the behaviour with changing limb darkening $\epsilon$ for rigid rotation $(\alpha=0)$; in Fig. 3 we consider variable amounts of differential rotation for constant $\epsilon=0.6$; and in Fig. 4 we investigate the influence of varying the inclination angles $i$ for the case of a solar-like differentially roting star $(\alpha=0.35)$ with $\epsilon=0.6$.

In the data domain all three parameters $\alpha, \epsilon$ and $i$ change the line profile in a similar way, also the changes are at the percent level showing the necessity of high signalto-noise data. In Fourier domain, however, the signatures become distinguishable; note that in the Fourier domain the ordinate is plotted with a logarithmic scale, while in 

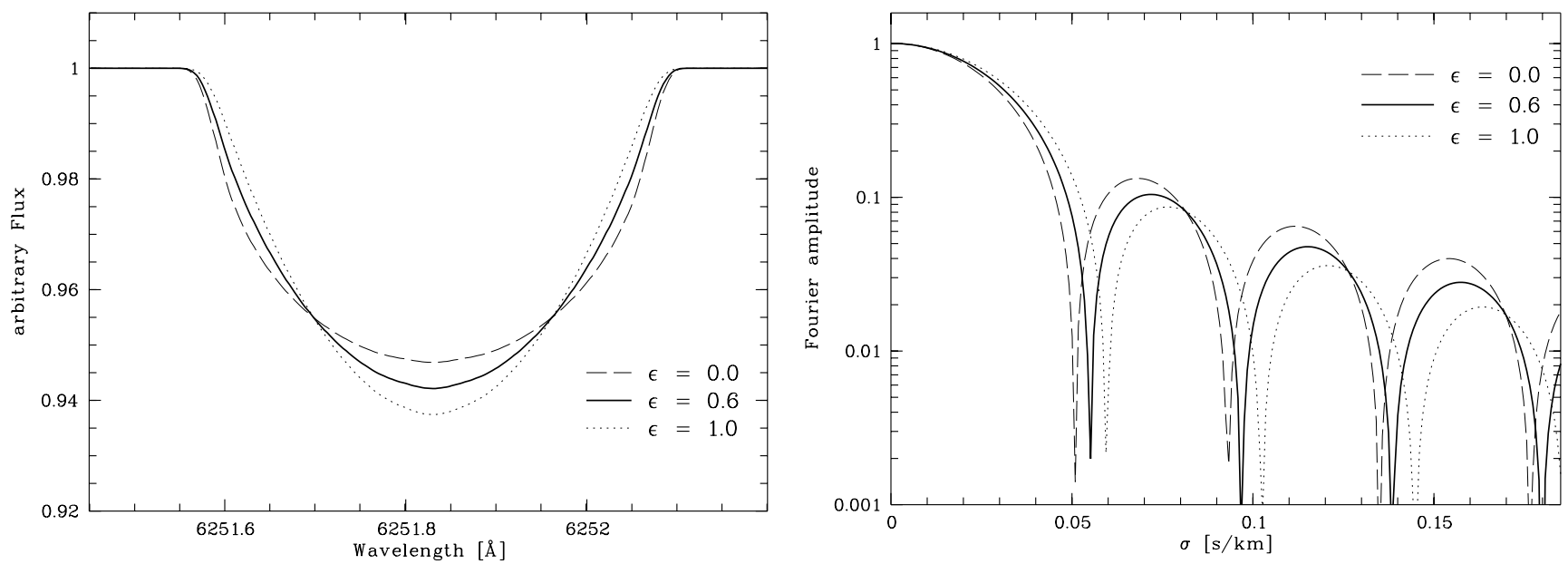

Fig. 2. Absorption line profiles for $v \sin i=12 \mathrm{~km} \mathrm{~s}^{-1}$ and rigid rotation $\left(\alpha=0 ; i=90^{\circ}\right)$ in data domain (left) and Fourier domain (right). Three different cases of limb darkening $(\epsilon=0.0, \epsilon=0.6$ and $\epsilon=1.0)$ are indicated by dashed, solid and dotted lines, respectively. Note that in Fourier domain the ordinate is plotted with logarithmic scale, while in data domain it is a linear scale.
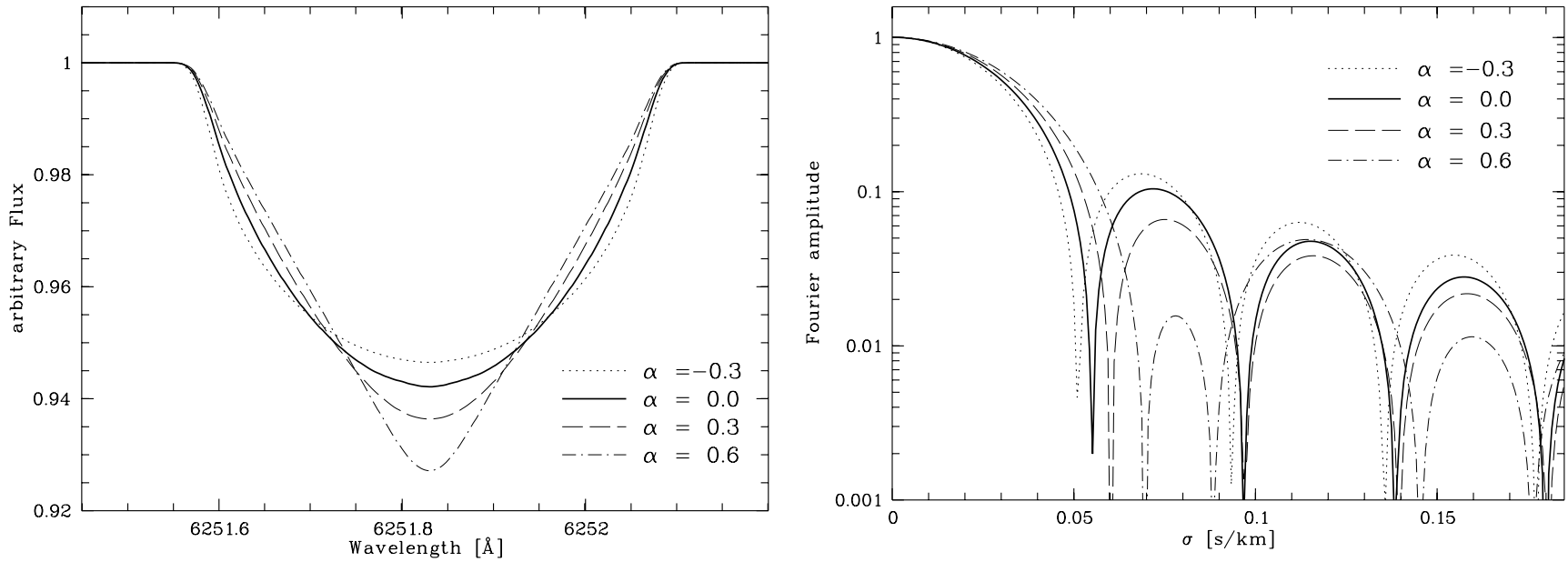

Fig. 3. Absorption line profiles as in Fig. 2 for limb darkening $\epsilon=0.6$ and $i=90^{\circ}$. Different cases of differential rotation ( $\alpha=-0.3, \alpha=0.0, \alpha=0.3$ and $\alpha=0.6$ ) are indicated. In the Fourier domain the different behaviour of the first sidelobe is evident, it narrows for larger differential rotation, while it's amplitude lessens. The amplitude of the second sidelobe changes slightly.
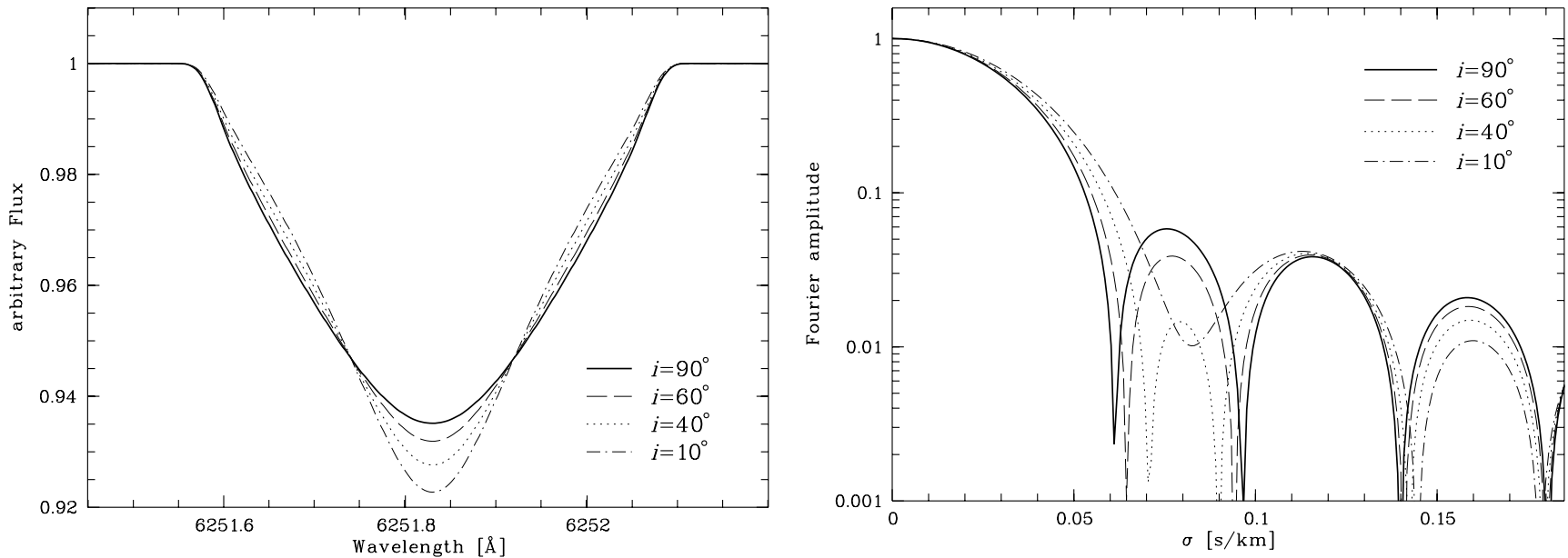

Fig. 4. Absorption line profiles as in Fig. 2 for limb darkening $\epsilon=0.6$ and differential rotation $(\alpha=0.35)$. Different inclination angles at constant $v \sin i\left(i=90^{\circ}, i=60^{\circ}, i=40^{\circ}\right.$ and $\left.i=10^{\circ}\right)$ are indicated. The behaviour of the first sidelobe with smaller inclination is comparable to the case of larger differential rotation. The amplitude of the second sidelobe remains constant. 

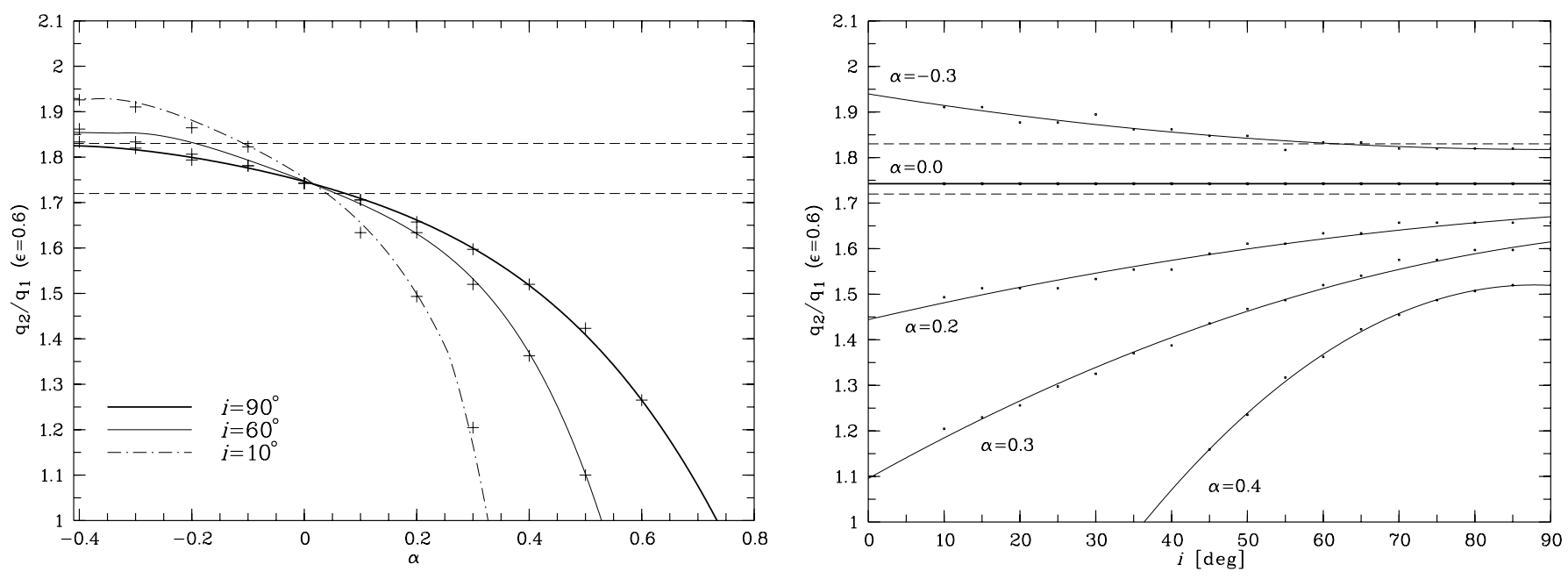

Fig. 5. $q_{2} / q_{1}$ plotted versus $\alpha$ (left) and versus $i$ (right); $\epsilon=0.6$. Calculated values are marked by crosses in the left and by dots in the right plot. Hand drawn lines of constant inclination (left) respectively constant differential rotation (right) connect the values. Dashed lines define the region which can be achieved by rigid rotation $(\alpha=0.0)$ and varying limb darkening $(0.0 \leq \epsilon . \leq 1.0)$.

the data domain it is a linear scale. We confirm that limb darkening (Fig. 2) changes the zero positions $\sigma_{i}$ and amplitudes of all sidelobes $I_{i}$ in a similar way. Differential rotation (Fig. 3) narrows the first sidelobe and diminishes its amplitude $I_{1}$ while the amplitude of the second sidelobe $I_{2}$ is only slightly affected. Our calculations are consistent with Gray (1977), Bruning (1981) and GarcíaAlegre et al. (1982) and confirm that the first sidelobe of a Fourier transformed line profile is sensitive to differential rotation. As can be seen in Fig. 4, smaller inclination angles do mimic stronger differential rotation; note that $v \sin i$ remains constant in the profiles. For inclination angles as small as $i=10^{\circ}$ the first sidelobe even vanishes. On the other hand, the amplitude of the second sidelobe is only slightly affected by changing differential rotation $\alpha$ (non-varying inclination $i$, Fig. 3) and it remains almost constant with changing inclination $i$ and constant differential rotation $\alpha$ (Fig. 4).

Bruning (1981) calculated $\sigma_{1}, \sigma_{2}$ and $I_{1}$ while GarcíaAlegre et al. (1982) showed $I_{1}$ and $I_{1} / I_{2}$. Our results are in good agreement with the results of Bruning for $i=90^{\circ}$, for $i<90^{\circ}$ deviations of up to $15 \%$ can be recognized. Calculations of $I_{1}$ agree with the calculations from GarcíaAlegre et al. for all cases of $\alpha$ and $i$ while their ratios of first and second sidelobe amplitudes $I_{1} / I_{2}$ are systematically higher (up to 25\%) than our values. We attribute these differences to the width of the used input function. García-Alegre et al. used a Gaussian profile which is not further specified. If their input function has a significant line width, the amplitudes of higher sidelobes will be diminished, as explained in Sect. 3 resulting in a higher ratio $I_{1} / I_{2}$.

The two most instructive ratios of observable parameters are, first, the ratio of the second and first zero positions $\sigma_{2} / \sigma_{1}$ (which is identical to $q_{2} / q_{1}$ ) and, second, the ratio of the first and second sidelobe amplitudes $I_{1} / I_{2}$.
According to Eq. (11) the value of $q_{2} / q_{1}$ varies between 1.72 and 1.83 for a rigidly rotating star by varying $\epsilon$. As mentioned in Sect. 2, for a sufficiently high rotation rate the zero positions of a Fourier transformed profile are only affected by the rotation law. A measured value of $\sigma_{2} / \sigma_{1}$ outside that range $(1.72-1.83)$ must therefore be a direct indication of differential rotation.

Figure 5 shows the value $q_{2} / q_{1}$ for different combinations of $\alpha$ and $i$ (keeping $\epsilon=0.6$ fixed). In the left panel the values are plotted versus $\alpha$, in the right panel versus inclination $i$. Calculated values are marked by crosses in the left and by dots in the right panel. Lines of constant inclinations (left) resp. constant differential rotation values (right) connect the calculated values. The dashed lines define the region of $q_{2} / q_{1}$ between 1.72 and 1.83 , where rigid rotation is possible.

Clearly, well defined dependences of $q_{2} / q_{1}$ on $\alpha$ and $i$ appear which exceed the effects caused by limb darkening and rigid rotation alone. In all cases $q_{2} / q_{1}$ shows a monotonic behaviour; it diminishes (the first sidelobe becomes narrower) with larger $\alpha$ and smaller inclination $i$. For extreme values of differential rotation and for small inclination angles, $q_{2} / q_{1}$ crosses the ordinate, i.e. the first sidelobe vanishes (cf. Fig. 4). Although it is not possible to determine both $\alpha$ and $i$ from a measured value of $q_{2} / q_{1}$ simultaneously, this easily measured value is suitable to rule out rigid rotation for many cases.

Figure 6 shows a contour plot of the parameter $q_{2} / q_{1}$ vs. $\alpha$ and $i$ for the case $\epsilon=0.6$ (solid lines); it can be used to determine the permitted $\alpha / i$-combinations from a measured $q_{2} / q_{1}$-ratio. The dashed lines mark the values of $q_{2} / q_{1}$ which can be achieved with solid body rotation and extreme limb darkening parameters alone. Small values of $q_{2} / q_{1}$ are expected for small inclination angles combined with differential rotation of the order of the solar value. Above the 1.3-contour the gradient of $q_{2} / q_{1}$ is very steep 


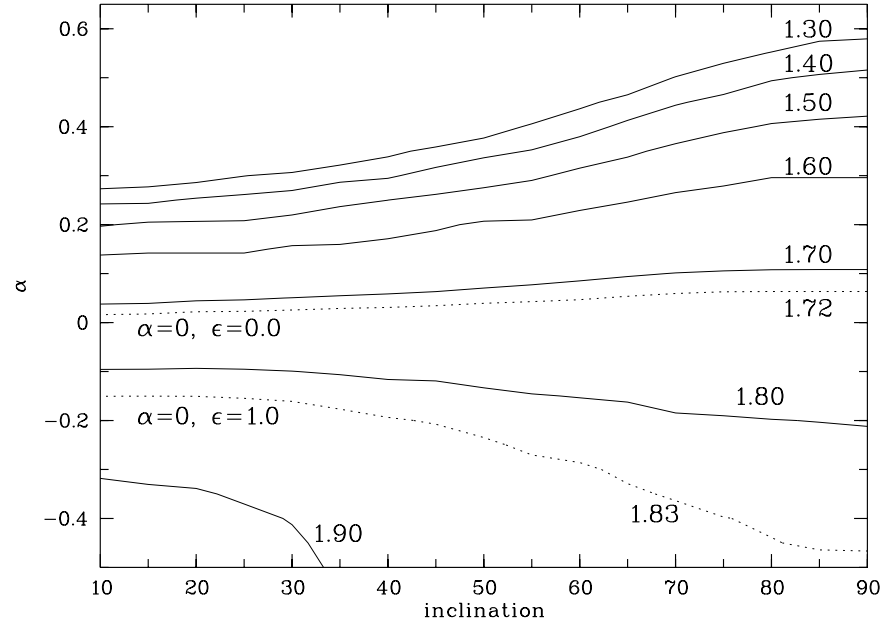

Fig. 6. Contour-plot of $q_{2} / q_{1}$ in the $\alpha-i$ plane. Solid lines mark the possible combinations of differential rotation $\alpha$ and inclination $i$ for a given $q_{2} / q_{1}$ with limb darkening $\epsilon=0.6$. Dashed lines show the region which is accessible with rigid rotation $(\alpha=0.0)$ and varying limb darkening $\epsilon$.

and lines for $q_{2} / q_{1}=1.2$ and 1.1 are not drawn for better legibility. The first sidelobe vanishes close to the 1.3-line.

The second measurable ratio, $I_{1} / I_{2}$, is shown in Fig. 7 for $\epsilon=0.6$. A systematic behaviour can also be noticed here; for positive $\alpha$ (solar-like differential rotation) the situation is comparable with that of $q_{2} / q_{1}$, but for negative $\alpha$ (anti-solar differential rotation; polar regions rotate faster than the equator) the ratio lessens as well. Thus $I_{1} / I_{2}$ can validate results obtained with $q_{2} / q_{1}$, but the sense of differential rotation cannot be determined using this value. Moreover, unlike $q_{2} / q_{1}, I_{1} / I_{2}$ is affected by the intrinsic line profile $f(\sigma)$, which involves additional velocity fields, intrinsic parameters like temperature and atomic data, and the instrumental profile. In Fig. 1 the shown Fourier transform of the isotropic velocity field with $v_{\text {iso }}=5 \mathrm{~km} \mathrm{~s}^{-1}$ can be interpreted as an approximation of a Fourier transformed intrinsic line profile $f(\sigma)$. While $g(\sigma)$ scales in $\sigma$-direction with varying $v \sin i, f(\sigma)$ remains constant. Thus the sidelobe amplitudes of $d(\sigma)$ are affected by the shape of $f(\sigma)$ and particularly for slower rotators $I_{1} / I_{2}$ becomes unreliable. Therefore, in order to use the ratio $I_{1} / I_{2}$ for determination of differential rotation, $g(\sigma)$ has to be deconvolved from the data $d(\sigma)$, and especially for slower rotators a complete line profile modelling is required.

\section{An example: $\psi$ Cap}

As an example for the application of the above procedure we show the determination of $\alpha / i$-combinations from the absorption line Fe I $\lambda 5775$ of the differentially and rapidly rotating $\mathrm{F} 5$ dwarf $\psi \operatorname{Cap}\left(v \sin i=42 \pm 1 \mathrm{~km} \mathrm{~s}^{-1}\right)$. The data has been taken during an $810 \mathrm{~s}$ exposure on Oct. 13, 2000 with the CES at ESO $3.6 \mathrm{~m}, S / N \sim 800$; a complete analysis has been presented by Reiners et al. (2001). Although the $S / N$ ratio is rather high, we mirrored the
Table 1. Fourier transformed Fe I $\lambda 5775$ intensities and zero positions of $\psi$ Cap.

\begin{tabular}{|c|c|c|c|c|c|c|}
\hline & $q_{1}$ & $q_{2}$ & $I_{1}$ & $I_{2}$ & $q_{2} / q_{1}$ & $I_{1} / I_{2}$ \\
\hline & 0.017 & 0.027 & 0.078 & 0.041 & 1.65 & 1.914 \\
\hline \pm & 0.001 & 0.001 & 0.003 & 0.003 & 0.01 & 0.004 \\
\hline
\end{tabular}

line profile at its center to achieve an even higher $S / N$ ratio and to obtain a symmetric profile. Since the main broadening mechanism for $\psi$ Cap is rotation, symmetry of the profile is expected and no problems should arise with mirroring. No further corrections were applied to the data and especially no corrections for turbulence or instrumental broadening were made.

In Table 1 we show the values for zero positions and sidelobe intensities derived from our CES spectrum of $\psi$ Cap. The zero positions were determined from the intersection of a regression line defined by the nearest three points neighbouring the zero position with unambigious sign and the abscissa. We estimated the error of the zero positions by regression lines through the respective point plus (minus) $S_{\sigma}$. For $\psi$ Cap we derive $q_{2} / q_{1}=1.65 \pm 0.01$. In Fig. 8 the corresponding combinations of inclination $i$ and differential rotation $\alpha$ is shown. Clearly, the thus defined region (black area with $1.64 \leq q_{2} / q_{1} \leq 1.66$ ) is significantly distinct from that accessible by rigid rotation and arbitrary limb darkening. In particular, the thus defined region is fully consistent with the parameter region determined from a complete atmospheric modelling of the $\psi$ Cap data (cf. Fig. 4 in Reiners et al. 2001). Since the result derived from $q_{2} / q_{1}$ is the same as that derived from the complete atmospheric fit, we argue that $q_{2} / q_{1}$ is an adequate observable to quickly and reliably determine solar-like $(\alpha>0)$ differential rotation for fast rotators.

We mention in passing that the measured ratio of the sidelobe amplitudes $I_{1} / I_{2}=1.914 \pm 0.004$ also supports the result that $\psi$ Cap is no rigid rotator (cp. with Fig. 7). Although we showed that great care has to be taken using $I_{1} / I_{2}$ for differential rotation determination, in the case of the fast rotator $\psi$ Cap the sidelobe amplitudes are expected to be only marginally affected by turbulent velocity fields and instrumental effects.

\section{Summary}

We have carried out detailed calculations of the dependence of Fourier transformed line profiles on differential rotation $\alpha$ and the inclination angle, focusing on the question, to what extent differential rotation can be distinguished from limb darkening effects and to what extent inclination matters. We have excluded the question of how starspots can influence the reliability of the method. This will be the topic of a further publication.

Our calculations assume an approximation of the Maunder differential rotation law analogous to that derived for the solar case and a linear limb darkening law. Alternative rotation or limb darkening laws and influences 

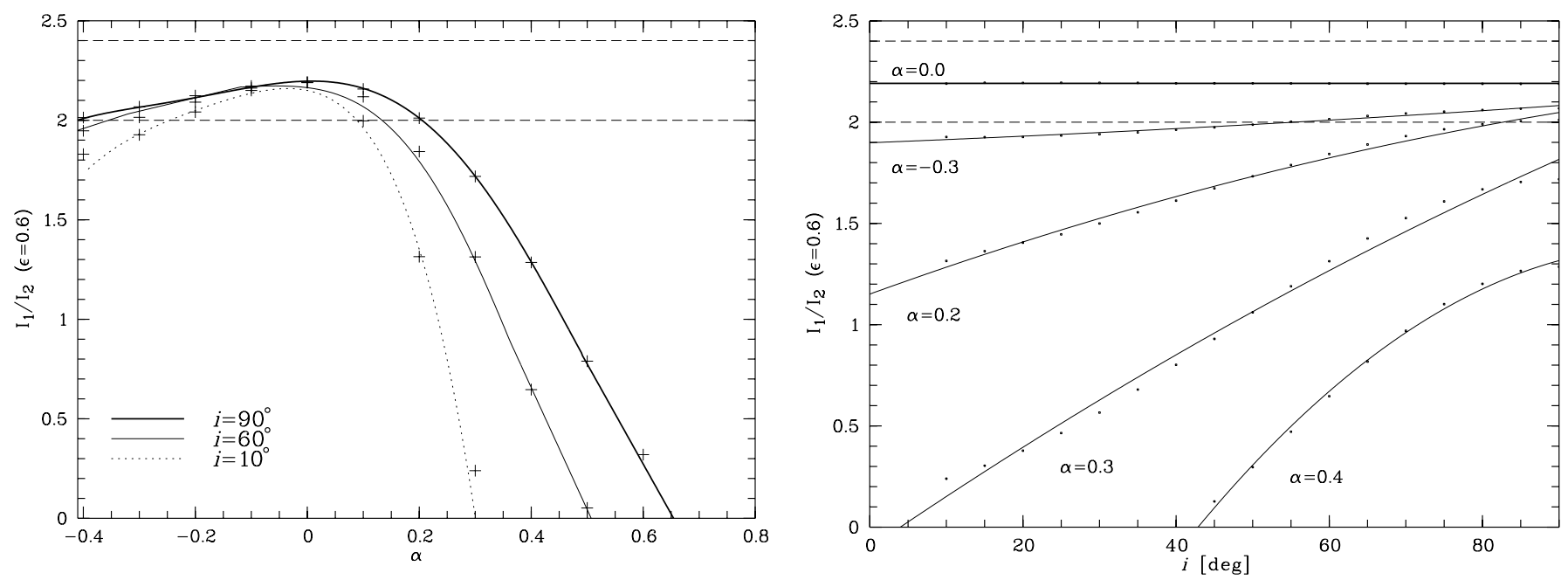

Fig. 7. $I_{1} / I_{2}$ for $\epsilon=0.6$ plotted in the same way as $q_{2} / q_{1}$ in Fig. $5 . I_{1} / I_{2}$ also depends strongly on $\alpha$ and $i$ but shows a non-monotonic behaviour. Note that in the right panel the line for $\alpha=-0.3$ lies below the line for $\alpha=0.0$.

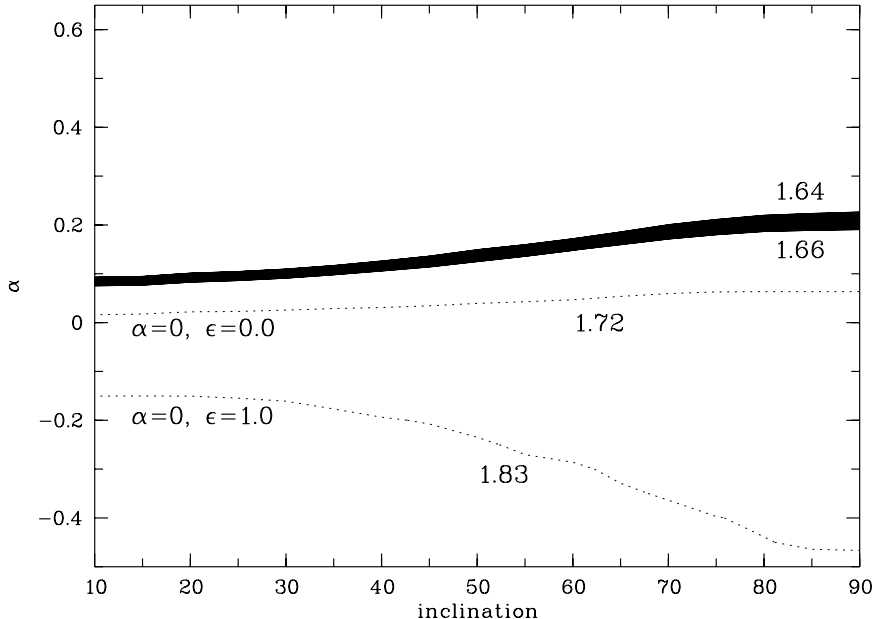

Fig. 8. Contour-plot of $q_{2} / q_{1}$ with the derived region for $\psi$ Cap $\left(1.64 \leq q_{2} / q_{1} \leq 1.66\right)$ marked black. The black area is clearly different from that accessible with rigid rotation (area between the dottet lines) and occupies the same region as that found by Reiners et al. (2001) in their Fig. 4.

of spots have not been investigated yet. Although we intend to carry out calculations including a greater variety of assumptions we do not expect large differences in our results. Our analysis focuses on the low frequency part of the Fourier transforms while small bumps produced by small scale spots or small deviations from the rotation and limb darkening laws are expected to influence only the high frequency part of the Fourier spectrum. However, we do want to point out that large polar spots as found in many Doppler images on a variety of stars may possibly influence our results.

Two measurable values $-q_{2} / q_{1}$, the ratio of the second and the first zero of the Fourier transform, and $I_{1} / I_{2}$, the ratio of the amplitudes of the first and second sidelobes have been studied. A reliable interpretation of a measured

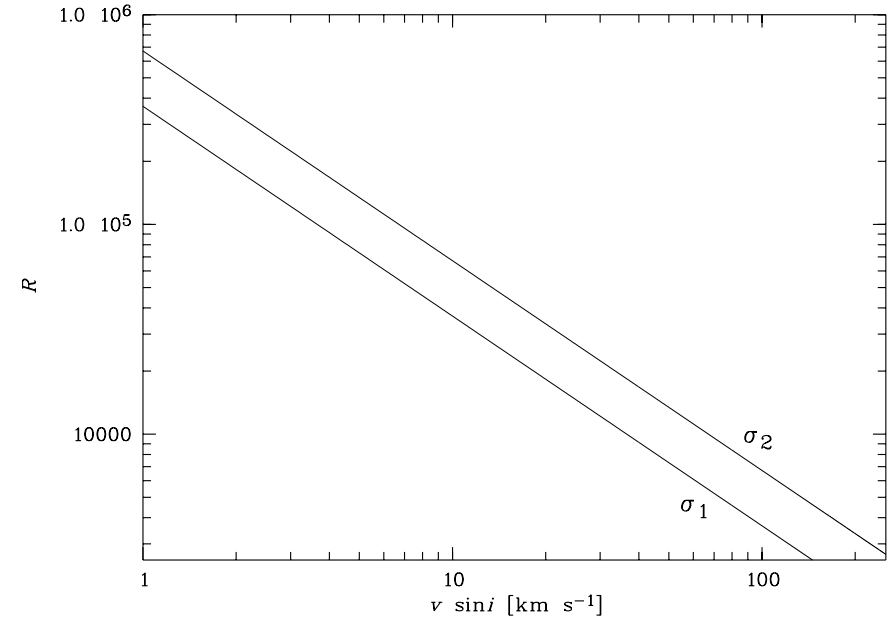

Fig. 9. The required resolution $R$ for the detection of the first $\left(\sigma_{1}\right)$ and second $\left(\sigma_{2}\right)$ zero positions for a star with a projected rotational velocity of $v \sin i$ and rigid rotation $(\alpha=0.0)$.

value of $I_{1} / I_{2}$ is quite difficult because those ratios are affected by the - in general unknown - intrinsic line profile. Only for very rapid rotators this ratio can be disentangled from the intrinsic line profile characteristics. The sign of differential rotation cannot be determined with this ratio. However, $q_{2} / q_{1}$ turned out to be a very reliable tracer of differential rotation. The measurement of $q_{2} / q_{1}$ is straightforward and can be used without any modelling of line profiles. $q_{2} / q_{1}$ does carry information about differential rotation; a value of $q_{2} / q_{1}<1.72$ is a direct indication for a solar-like differential rotation law, while $q_{2} / q_{1}>1.83$ indicates anti-solar differential rotation. The combination of inclination angle $i$ and differential rotation $\alpha$ remains ambiguous, but information on period and radius of the star can confine possible parameter regions. 
As is clear from Fig. 6 for a given value of $\alpha$, smaller inclinations always lead to larger deviations from the rigid rotation case, but obviously small inclination angles diminish the projected value of $v \sin i$ and the given spectral resolution limits the measurement of $\sigma_{2}$. Consequently sufficiently large $v \sin i$ values are needed. Thus there is a bias in our detectability of differential rotation.

For slow rotators the Nyquist frequency $\sigma_{\nu}$, i.e. the maximum Fourier frequency $\sigma$ contained in a Fourier transform of a line profile obtained with a resolution $R$, limits the detection of $\sigma_{2}$. For fast rotators the situation improves dramatically and no problems arise with the measurement of $\sigma_{2}$. In Fig. 9 the required resolution for the detection of the first $\left(\sigma_{1}\right)$ and second $\left(\sigma_{2}\right)$ zero positions of $g(\sigma)$, the Fourier transform of a rigid rotation broadening function $G(\lambda)$, observed with a specified value of $v \sin i$ is shown. A resolution of the order $R=70000$ is needed to determine $\sigma_{2}$ for a star with $v \sin i=10 \mathrm{~km} \mathrm{~s}^{-1}$. Known complications like aliasing emphasize the need for a somewhat higher resolution.

Our picture of stellar rotation law is still very poor (e.g. Gray 1977, 1982; Wöhl 1983). Information on a large sample of moderate rotators with high sensitivity to differential rotation would be instructive for our understanding of stellar dynamo processes. This can easily be accomplished by measuring the $q_{2} / q_{1}$ ratios as shown in this paper.

\section{References}

Barnes, J. R., Collier Cameron, A., James, D. J., \& Donati, J.-F. 2000, MNRAS, 314, 162

Böhm, K. 1952, Zeit. f. Astrophysik, 30, 117

Bruning, D. H. 1981, ApJ, 248, 274

Bruning, D. H. 1982, A\&A, 115, 203

Carroll, J. A. 1933a, MNRAS, 93, 478

Carroll, J. A. 1933b, MNRAS, 93, 680

Donati, J.-F., \& Collier Cameron, A. 1997, MNRAS, 291, 1

Dravins, D., Lindegren, L., \& Torkelsson, U. 1990, A\&A, 237, 137

García-Alegre, M. C., Vázquez, M., \& Wöhl, H. 1982, A\&A, 106, 261

Gray, D. F. 1976, The observation and analysis of stellar photospheres (Wiley, New York)

Gray, D. F. 1977, ApJ, 211, 198

Gray, D. F. 1982, ApJ, 258, 201

Huang, S.-S. 1961, ApJ, 133, 130

Kitchatinov, L. L., \& Rüdiger, G. 1999, A\&A, 344, 911

Reiners, A., Schmitt, J. H. M. M., \& Kürster, M. 2001, A\&A, 376, L13

Townsend, R. 1997, MNRAS, 284, 839

Unsöld, A. 1968, Physik der Sternatmosphären (Springer, Berlin)

Weber, M., \& Strassmeier, K. G. 2001, A\&A, 373, 974

Wöhl, H. 1983, in Solar and Stellar Magnetic Fields: Origin and Coronal Effects, ed. J. O. Stenflo (Reidel Dordrecht), IAU Symp., 102, 155 